\title{
Assessment of autonomic nervous system functions and cardiac rhythms in patients using isotretinoin
}

\author{
Halil Ay ${ }^{1}$, Mustafa Aksoy², Fatih Güngören ${ }^{3}$ \\ 'Department of Neurology, Harran University, Şanlıurfa, Turkey \\ 2Department of Dermatology, Harran University, Şanlıurfa, Turkey \\ ${ }^{3}$ Department of Cardiology, Harran University, Şanlıurfa, Turkey
}

Adv Dermatol Allergol 2019; XXXVI (3): 295-298

DOI: https://doi.org/10.5114/ada.2018.76848

\begin{abstract}
Aim: The aim of the study was to analyze the potential effects of isotretinoin, frequently used in the treatment of acne vulgaris these days.

Material and methods: For this purpose, we used the methods of sympathetic skin response (SSR) electrophysiological analysis and electrocardiographic (ECG) analysis in patients using isotretinoin. Thirty patients who were diagnosed with acne vulgaris and treated with oral isotretinoin with a dose of $0.5 \mathrm{mg} / \mathrm{kg}$ for at least 1 month were included in the study. In all patients ECG scanning and SSR analysis were performed both before treatment and 1 month after the start of treatment.

Conclusions: This study is very important because SSR results show that increasing the existing sympathetic activity in acne vulgaris after isotretinoin usage could explain the exacerbation in acne lesions for the first month, and according to the ECG results the medication did not cause cardiac side effects.
\end{abstract}

Key words: isotretinoin, sympathetic skin response, acne vulgaris.

\section{Introduction}

Isotretinoin, the most effective medication in acne treatment these days, exerts its effects through four pathogenic factors. These factors can be summarized as that the medication affects the progression of the cell cycle, cellular differentiation, cell recovery and apoptosis. In this way it causes a significant decrease in sebum production, affects comedogenesis, decreases surface and ductal Propionibacterium acnes, and demonstrates anti-inflammatory effects. Isotretinoin is recommended in treatment of serious forms such as nodules or conglobate acne, when there is a risk of permanent scar formation and when standard treatment with systemic antibiotics and topical treatment are not satisfactory [1].

Isotretinoin is significantly effective; however, it is associated with many side effects which can restrict its usage. The main adverse effect of isotretinoin is known as teratogenicity. Despite the fact that all of them are very rare, the other side effects can be listed as cheilitis, dryness of eye and mouth, photosensitivity, conjunctivitis, hypertriglyceridemia, pancreatitis, increase of serum cholesterol levels and liver enzymes, blood dyscrasia, hy- perostosis, early epiphysis closure and nyctalopia [2]. In many recent studies there were no determined negative effects of isotretinoin on cardiac functions $[3,4]$.

However, there has been no recent study researching the potential effects of isotretinoin on the autonomic nervous system by carrying out SSR analysis.

The sympathetic skin response (SSR) is defined as the electrical potential change occurring on skin after stimulation with any internal or external stimulus. This reflex, which can be stimulated in several ways, uses preganglionic and postganglionic sympathetic sudomotor fibers as an efferent pathway. It reaches the last organ, the sweat glands [5]. It was asserted that the SSR represents sympathetic sudomotor flow in the central and peripheral nervous system. Recently many studies have indicated a potential relation between SSR and autonomic dysfunction, and there are even significant changes in response amplitude and latencies [6-8].

\section{Aim}

In this study the effects of the medication on the autonomic nervous system, heart rate and cardiac rhythm

Address for correspondence: Assist. Prof. Halil Ay, Department of Neurology, Harran University, 63050 Şanlıurfa, Turkey, phone: +90 5306934206, e-mail: ayhalil27@hotmail.com Received: 9.04.2018, accepted: 22.05.2018. 
were investigated by carrying out SSR analysis and ECG scanning in patients admitted to the dermatology clinic due to acne vulgaris.

\section{Material and methods}

\section{Materials}

Thirty patients diagnosed with acne vulgaris were included in the study. The average age of the patients was $20.93 \pm 5.03(21.04 \pm 4.93$ for women; $20.62 \pm 5.62$ for men). Twenty-two (77.3\%) of the patients were women and $8(26.7 \%)$ were men. In all patients ECG scanning and SSR analysis were performed both before treatment and 1 month after the start of treatment.

None of the patients had any other diseases apart from acne vulgaris. They had received treatment with oral isotretinoin at a dose of $0.5 \mathrm{mg} / \mathrm{kg}$ per day for at least 1 month. Patients with diseases such as cardiac disease, diabetes mellitus, hypo/hyperthyroidism, chronic obstructive respiratory disease, congestive cardiac failure, coronary artery disease, alcoholism, collagen tissue disease, atrioventricular transmission abnormalities and people on medications known to change cardiac transmission were excluded from the study.

From all patients informed consent forms were obtained, and before the beginning of the study ethics committee approval was obtained from Harran University.

\section{Electrophysiological study}

In all patients SSR analysis was performed before and 1 month after treatment. In our electromyography (EMG) unit SSR analysis was carried out before and 1 month after treatment using Dantec Keypoint EMG Device V2.32. SSR analysis was recorded by placing active electrodes into the palm and surface electrodes on the dorsum of the hand after cleaning the skin of hand and wrist while the patient was lying down. For recording, normal ambient temperature $\left(24-26^{\circ} \mathrm{C}\right)$ was used and the temperature of the hand skin was $30^{\circ} \mathrm{C}$, and when necessary the patient was warmed up. Silver cup electrodes were used as recording electrodes.

In SSR analysis rapid development of habituation after consecutive electrical stimulations is a well-known rule [9]. Therefore, electrical stimulation was applied to the contralateral median nerve in total 4 times with at least a 3-minute interval, thereby avoiding habituation. The average of these four stimulations was calculated. During recording the filter setting was between $0.5 \mathrm{~Hz}$ and $2 \mathrm{kHz}$, stimulation time was $0.01 \mathrm{~s}$ and the stimulation level was $25 \mathrm{~mA}$. SSR latency was measured by taking the negative deflection starting point into account, whereas amplitude of SSR was measured by taking peak points of both negative and positive deflections into account.

\section{Electrocardiographic study}

In all patients ECG analysis was carried out before and 1 month after treatment. Heart rate, P-wave time, PR distance, PR segment, QRS time, T-wave time, QT distance, QTc distance, T peak-end (Tpe), T peak-end/ QT (Tpe/QT), and T peak-end/QTc (Tpe/QTc) parameters were observed.

\section{Arterial tension measurement}

In all patients systolic and diastolic arterial tension measurement was performed before and 1 month after treatment.

\section{Statistical analysis}

Statistical analysis was performed using the SPSS 20.0 one-way ANOVA post hoc Tukey test.

\section{Results}

In patients SSR analysis was carried out before and 1 month after treatment. Prior to treatment average SSR latency was $1506.43 \pm 246.20 \mathrm{~ms}$, whereas SSR latency average after treatment was $1275.03 \pm 170.71$. The SSR latencies after treatment were obviously shorter than those before treatment and the difference was statistically significant $(p<0.001)$ (Figure 1 ).

Before treatment the average SSR amplitude was $2.69 \pm 1.68 \mathrm{mV}$. After treatment the average SSR amplitude was $2.27 \pm 1.80 \mathrm{mV}$. The SSR amplitude after treatment was slightly lower, but the difference was not statistically significant ( $p=0.365$ ).

In patients ECG scanning was performed before and 1 month after treatment. Before treatment the heart rate was $85.21 \pm 15.59$ beats $/ \mathrm{min}$, but after treatment it was $81.07 \pm 15.63$ beats $/ \mathrm{min}$. No significant difference was found when QTc distance, Tpe, Tpe/QTc values and other ECG parameters, which are predictors of pre-treatment malign cardiac arrhythmia, were compared with the values obtained after treatment. All ECG parameters are shown in detail in Table 1. Moreover, pre-treatment systolic blood pressure was $111.78 \pm 9.83 \mathrm{~mm} \mathrm{Hg}$, but it was $112.77 \pm 10.40 \mathrm{~mm} \mathrm{Hg}$ after treatment. Pre-treatment diastolic blood pressure was $68.21 \pm 7.72 \mathrm{~mm} \mathrm{Hg}$, but it was $70.92 \pm 8.55 \mathrm{~mm} \mathrm{Hg}$ after treatment. No significant difference was found in the blood pressure values before and after treatment $(p>0.05)$.

\section{Discussion}

Acne vulgaris is a chronic inflammatory disease of the pilosebaceous unit, and it occurs in skin regions where the most sebaceous glands exist. In 99\% of patients it affects the face [10]. Although it predominantly affects the adolescent population (approximately 85\%), pre- and post-adolescents can also be affected [11, 12]. Although 
there are several treatment options for acne vulgaris, only isotretinoin has an influence with effects on all primary etiological factors [13]. But in most of the patients an increase is observed in acne lesions for the first month [14].

Until now there has been no study researching the effects of isotretinoin (used in the treatment of acne vulgaris) in the autonomic nervous system by carrying out SSR analysis. In this study we aimed to reveal both negative and positive effects of isotretinoin in acne vulgaris treatment by examining the effects of the medication in autonomic and cardiac functions apart from the potential effects of it by performing SSR analysis and ECG scanning in subjects.

Skin is innervated with sensory nerves, postganglionic sympathetic and parasympathetic nerve fibers. In general, it is accepted that sebaceous glands are not innervated by the peripheral nervous system. However, it was reported that the number of nerve fibers around sebaceous glands in facial skin is high in acne vulgaris patients, whereas it was found that nerve fibers in normal facial skin were rare [15].

In a study that included 50 subjects with atopic dermatitis and 50 healthy subjects conducted by Wruhs et al., sudomotor activity (activity of sweat glands) in the two groups was measured by the SSR method [15]. It was found that the average SSR latency was significantly longer in the atopic group than the control group. Average SSR amplitude was lower in the atopic group than the control group, but the difference was not statistically significant. Wruhs et al. postulated that extension of SSR latency may be an indication of sympathetic innervation deficiency [15]. The findings of Wruhs et al. suggest that, as in dermatomyositis or polymyositis, the nerve fibers within the related tissue are secondarily affected by inflammation. In parallel with the results of Wruhs et al., in our study pre-treatment SSR latencies were notably and statistically significantly longer than those after treatment. This situation recalls a sympathetic dysfunction in both atopic dermatitis and acne vulgaris.

Kaplan et al. observed that in patients with acne vulgaris for which thoracoscopic sympathectomy was performed, acne lesions remitted after sympathectomy [16]. During isotretinoin treatment, at the end of the first month of treatment, an increase in sympathetic activity can explain the exacerbation of acne lesions during the first month in most of the patients. However, when it is considered that normal isotretinoin treatment can take 20 weeks, if the SSR study had continued in months 2, 3, 4 and 5, probably SSR latencies would have been longer in the following months when compared to the first month. Therefore, we can assume that there was a role of isotretinoin in the sympathetic activity decrease for remission of acne lesions. Unfortunately, we could not achieve sufficient cooperation with the patients included in the study for a long-term study. Therefore, the study was planned as before treatment and 1 month after treatment.

The increase in sympathetic activity during the first month of isotretinoin treatment can be explained as follows. SSR is known to include suprasegmental inputs from

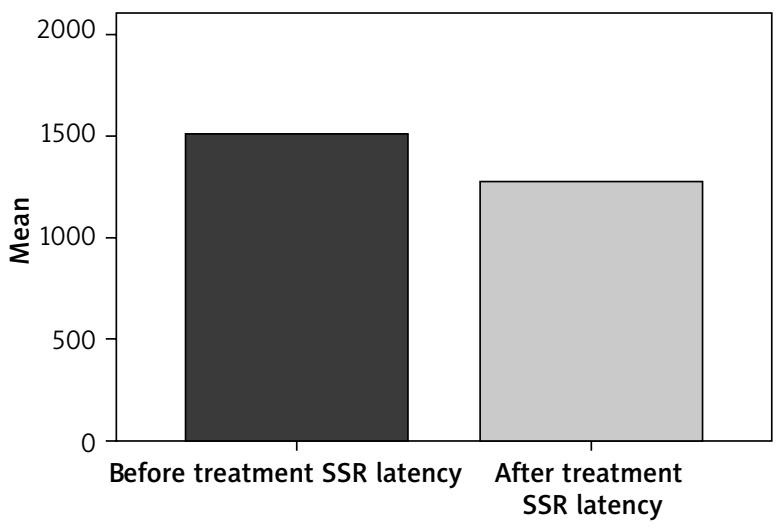

Figure 1. The SSR latencies before and after treatment

Table 1. ECG parameters

\begin{tabular}{lcc}
\hline Parameter & Before treatment & After treatment \\
\hline Heart rate [v/min] & $85.21 \pm 15.59$ & $81.07 \pm 15.63$ \\
\hline P wave duration [ms] & $67.8 \pm 17.5$ & $65.1 \pm 15.2$ \\
\hline PR distance $[\mathrm{ms}]$ & $128.2 \pm 18.2$ & $125.9 \pm 17.3$ \\
\hline PR segments [ms] & $60.3 \pm 17.7$ & $60.7 \pm 17.0$ \\
\hline QRS duration [ms] & $50.0 \pm 12.7$ & $54.0 \pm 14.4$ \\
\hline T dalga duration [ms] & $132.8 \pm 26.2$ & $129.6 \pm 21$ \\
\hline QT distance [ms] & $328.5 \pm 31.4$ & $333.7 \pm 29.2$ \\
\hline QTc distance [ms] & $385.7 \pm 29.4$ & $381.8 \pm 24.3$ \\
\hline Tpe [ms] & $64.2 \pm 20.4$ & $69.2 \pm 13.8$ \\
\hline Tpe/QTc & $0.16 \pm 0.05$ & $0.18 \pm 0.03$ \\
\hline
\end{tabular}

ms - milisecond, $v / m i n$ - velocity/minute.

the cerebral cortex and inhibitory inputs from the striatum and to reflect the activity of the posterior hypothalamus and brainstem reticular formation [17]. From this point of view, it can be surmised that by depressing the inhibitory inputs within isotretinoin striatum, suprasegmental structures, partially released, could increase the sympathetic activity just the same as in the increase of the deep tendon reflex in first motor neuron disease after elimination of inhibitory mechanisms. Another mechanism of increase in sympathetic activity by isotretinoin is that it can cause an increase in nerve growth factor (NGF). Nerve growth factor is the best categorized member of the neurotrophin family; it is vital for survival of peripheral sympathetic and sensory neurons and basal frontal cholinergic neurons within the central nervous system [18].

In our study, before treatment SSR amplitude average was $2.69 \pm 1.68 \mathrm{mV}$ and after treatment SSR amplitude average was $2.27 \pm 1.80 \mathrm{mV}$. After treatment SSR amplitudes were slightly lower compared to before treatment, but there was no significance in values statistically. When it is considered that isotretinoin causes apoptosis of se- 
baceous glands, it can be expected that we obtain a SSR response with lower amplitude than sebaceous glands atrophied by isotretinoin just the same as $\mathrm{M}$ and $\mathrm{N}$ response obtained from motor and sensory fibers of atrophied tissue in routine electrophysiological studies.

In 1992 Drory et al. classified 100 healthy subjects based on the supposition that electrophysiological differences may occur depending on age, and they carried out an SSR analysis and observed that latency values extend with aging, amplitude values decrease, or a response can be obtained. Nevertheless, it is not known which factors decrease or increase the amplitude values. It is beyond doubt that there is a need for further research on this topic [19]. The average age of our patients was $20.93 \pm 5.03$.

Although the effects of isotretinoin on many systems are well known, its effects on the cardiovascular system have rarely been reported. Some parameters in ECG can be used to predict potential arrhythmias. Yan et al. indicated that parameters such as Tpe time, Tpe/QT time, and Tpe/ QTc can be used to predict ventricular arrhythmia formation [20]. In a study conducted in 45 patients receiving isotretinoin treatment for 6 months due to acne vulgaris, Dursun et al. observed no difference in QTc and QT dispersion, which is an indicator of malign ventricular arrhythmia, and in these patients it was safe in terms of ventricular arrhythmia for 6 months of acne treatment [21]. Although rare, in the literature there are cases indicating that isotretinoin can cause ventricular extrasystole and atrial tachycardia attacks [22, 23].

In our study, P-wave time, PR distance, QRS wave time, QT time, QTc time, Tpe time, Tpe/QT time, and Tpe/QTc time of patients were assessed with ECG scanning before and one month after isotretinoin treatment. Moreover, patients were monitored for tension before and after treatment. It was observed that there was no significant change in basal ECG parameters and Tpe, Tpe/QT and Tpe/QTc times, which may be an indicator of malign ventricular arrhythmia before and after treatment. In our study, during one-month isotretinoin treatment no cardiac arrhythmia or electrocardiographic changes were observed in patients.

\section{Conclusions}

Quantitative changes in SSR and ECG parameters were measured in patients using isotretinoin due to acne vulgaris. As a result, this study showed that SSR results indicating that isotretinoin increases the existing sympathetic activity in acne vulgaris can explain the exacerbation in acne lesions for the first month and according to the ECG results the medication shows no cardiac side effects; therefore, this study is quite important.

\section{Conflict of interest}

The authors declare no conflict of interest.

\section{References}

1. Zouboulis CC, Bettoli V. Management of severe acne. Br J Dermatol 2015; 172: 27-36.

2. Kraft J, Freiman A. Management of acne. CMAJ 2011; 183 : 430-5.

3. Karadag AS, Gumrukcuoglu HA, Gunes Bilgili S, et al. Does isotretinoin therapy have any effects on electrocardiography, heart rate and blood pressure? J Dermatolog Treat 2012; 23: 168-71.

4. Selcoki Y, Gorpelioglu C, Turgut F, et al. Isotretinoin: is there any arrhythmic effect? Int I Dermatol 2008; 47: 195-7.

5. Ertekin C. Santral ve periferik EMG book, Otonom sinir sistemi. 2006; 884-909.

6. Ke JQ, Shao SM, Zheng YY, et al. Sympathetic skin response and heart rate variability in predicting autonomic disorders in patients with Parkinson disease. Medicine (Baltimore) 2017; 96: e6523.

7. Fusina S, Conte S, Bertolasi L, et al. Sympathetic skin esponse asymmetry in early stage idiopathic Parkinson's disease. Clin Neurophysiol 1999; 110: 358-66.

8. Zakrzewska-Pniewska B, Jamrozik Z. Are electrophysiological autonomic tests useful in the assessment of dysautonomia in Parkinson's disease? Parkinsonism Relat Disord 2002; 9: 179-83.

9. Ozkul Y, Ay H. Habituation of sympathetic skin response in migraine and tension type headache. Auton Neurosci 2007; 134: 81-4.

10. Nast A, Dreno B, Bettoli V, et al. European evidence-based (S3) guidelines for the treatment of acne. J Eur Acad Dermatol Venereol 2012; 26 (Suppl 1): 1-29.

11. Halvorsen JA, Stern RS, Dalgard F, et al. Suicidal ideation, mental health problems, and social impairment are increased in adolescents with acne: a population-based study. J Invest Dermatol 2011; 131: 363-70.

12. Marqueling AL, Zane LT. Depression and suicidal behavior in acne patients treated with isotretinoin: a systematic review. Semin Cutan Med Surg 2007; 26: 210-20.

13. Güngör E. Akne. Turk J Dermatol 2012; 6: 138-49.

14. Toyoda M, Nakamura M, Morohashi M. Neuropeptides and sebaceous glands. Eur J Dermatol 2002; 12: 422-7.

15. Wruhs M, Gleiß A, Steiner A, Sator P. Quantity and quality of sweating in atopic dermatitis. Arch Dermatol Res 2017; 309: 787-93.

16. Kaplan T, Gunduz O, Oznur B, Han S. Could thoracoscopic sympathicotomy for hyperhidrosis also improve acne vulgaris? Kardiochir Torakochir Pol 2014; 11: 264-7.

17. Drake ME. Clinical neurophysiology of the autonomic nervous system. Clin EEG 1995; 26: 133-4.

18. Levi-Montalcini R. The nerve growth factor 35 years later. Science 1987; 234: 1154-62.

19. Drory VE, Korezyn AD. Sympathetic skin response: age effect. Neurology 1993; 43: 1818-20.

20. Yan GX, Martin J. Electrocardiographic T wave: a symbol of transmural dispersion of repolarization in theventricles. J Cardiovasc Electrophysiol 2003; 14: 639-40.

21. Dursun R, Alpaslan M, Caliskan M, et al. Isotretinoin does not prolong QT intervals and QT dispersion in patientswith severe acne: a surprising finding for a drug with numerous side effects. J Drugs Dermatol 2011; 10: 710-4.

22. Hasdemir C, Sagcan A, Sekuri C, Ildizli M, Ulucan C, Ceylan C. Isotretinoin (13-cis-retinoic acid) associated atrial tachycardia. Pacing Clin Electrophysiol 2005; 28: 348-9.

23. Alan S, Ünal B, Yildirim. Premature ventricular contractions associated with isotretinoin use. An Bras Dermatol 2016; 91 : 820-1. 